\title{
Testing Microelectronic Biofluidic Systems
}

\author{
Hans G. Kerkhoff
}

University of Twente

\section{Editor's note:}

Biochips can fail because of a wide variety of reasons, ranging from electrical defects (shorts, opens, and so on) to material properties, unexpected fluidic flow patterns, and chemical or biological contamination. Read this article to find out how to detect and locate various types of faults in biochips.

-Krishnendu Chakrabarty, Duke University guaranteeing their quality was extremely difficult. This difficulty was due to passive mechanical structures, such as valves in pumps, which complicate observation, and a lack of knowledge about possible defects in the technologies used and resulting fluidic responses. Although engineers could use
According to the 2005 International Technology Roadmap for Semiconductors (http://public.itrs.net), the integration of emerging nondigital CMOS technologies will require radically different test methods, posing a major challenge for designers and test engineers. One such technology is microelectronic fluidic (MEF) arrays, which have rapidly gained importance in many biological, pharmaceutical, and industrial applications. The advantages of these systems, such as operation speed, use of very small amounts of liquid, on-board droplet detection, signal conditioning, and vast digital signal processing, make them very promising. However, testable design of these devices in a mass-production environment is still in its infancy, hampering their low-cost introduction to the market.

This article describes analog and digital MEF design and testing methods. It doesn't deal with the testing of pure fluidic systems that don't require electronics, although many impressive results have appeared in this area. It also doesn't go into inkjet modules, the most successful MEF systems until now.

Electronic fluidic systems started as discrete continuous-flow devices based on microelectromechanical systems (MEMS), sometimes assembled on double-sided PCBs. This category of microfluidics, analog microfluidics, uses components such as pumps, mixers, and flow sensors connected through channels. It quickly became clear that testing these systems and
Spice-based simulation models of found defects and perform indirect electrical measurements using, for example, capacitances of pure mechanical parts, testing was complicated and cumbersome. ${ }^{1}$

With the arrival of the fully electrically controlled flowFET (flow field-effect transistor), featuring the absence of any moving mechanical parts, a new era in analog MEF systems and testing began. Several published works deal with testable design and test of analog microfluidics with flowFETs. ${ }^{2}$ Around this time, a digital alternative in the form of a fluidic droplet appeared. In this fully electrically controlled technology, digital microfluidics, fluid transport is based on discrete droplets, using the electrowetting-on-dielectric (EWOD) principle. Digital microfluidics has interesting features including flexibility, scalability, and ease of monitoring and direction reconfigurability. Occurring defects, test time optimization, concurrent testing, and diagnostics in digital microfluidics have appeared in the literature, and this article will treat them in more detail. $^{3-6}$

Advanced applications combine electronics with fluidic modules to perform control, signal conditioning, and additional data processing. A state-of-the-art example is a (separate) digital-microfluidic chip for biomedical applications, on a PCB. ${ }^{7}$ The next logical step, the monolithic heterogeneous integration of fluidics and microelectronics, is also possible. ${ }^{8}$ Figure 1 shows a cross section of such an implementation. 


\section{Analog microfluidics}

The flowFET is a relatively young device and is simple in construction. Figure 2a shows a cross section of the device. It consists of two parts connected by anodic bonding, forming a fluidic channel. On a Pyrex wafer, electrodes are deposited, which are covered with an oxide layer, and coatings can be used to influence the zeta potential. ${ }^{8}$ The top of the device is constructed by micromachining. One requirement is that the fluid should contain ions to enable electro-osmotic flow (EOF). The flowFET's behavior is similar to that of a MOSFET, in that it applies electrode voltages to control a fluid instead of an electric flow. Double-diffused metal-oxide semiconductor transistors (DMOSTs) deliver the electrodes' relatively high voltages $(40+)^{2}$

Figure $2 \mathrm{~b}$ shows a photograph of optical testing of the flowFET's operation. Optical-detection methods are used for carrying out measurements as well as for testing. Optical detection requires transparency to observe the channels and the introduction of small fluorescent beads to trace the fluid. Normally, test engineers use a charge-coupled-device (CCD) camera and store its time data in a video recorder. With laser techniques, even a 3D image is feasible. From this data, fluid velocities as well as changes resulting from obstructions in the channels can be calculated and observed. Optical detection has also been valuable in investigating local fluid recirculation. The flowFET's bidirectional features make it suitable for fluidic routing as well as mixing.

The flowFET's construction allows identification of several possible catastrophic as well as parametric defects-for example, oxide pin holes, electrode opens and shorts, and oxide and channel variations. Also, from a fluidic viewpoint, problems such as leakage and particle jamming in a channel can occur. Clearly, the latter type of fault cannot be found in conventional electronics. One approach to determining the effects is to use finite-element-method (FEM)based accurate simulations of small fluidic modules such as flowFET fluidic crossings. Engineers can use the CoventorWare or CFD-ACE+ software tools for

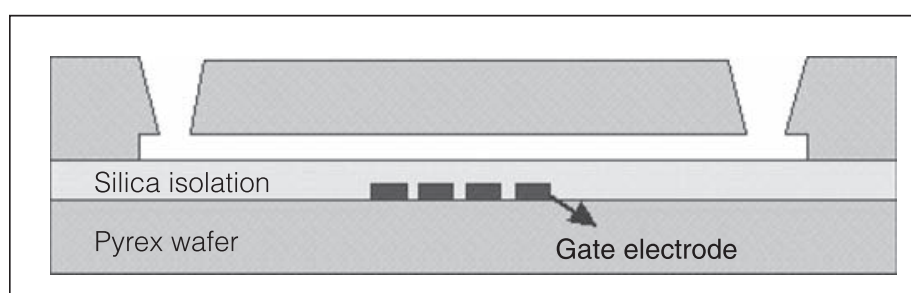

(a)

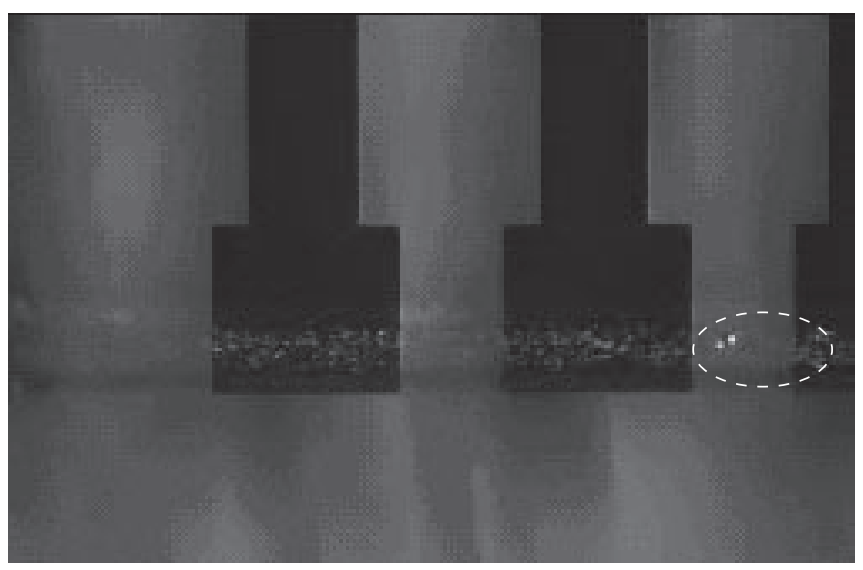

(b)

Figure 2. Cross section and construction of a flowfET (a) and optical testing of the flowFET flow using fluorescent beads (ellipse) (b). 


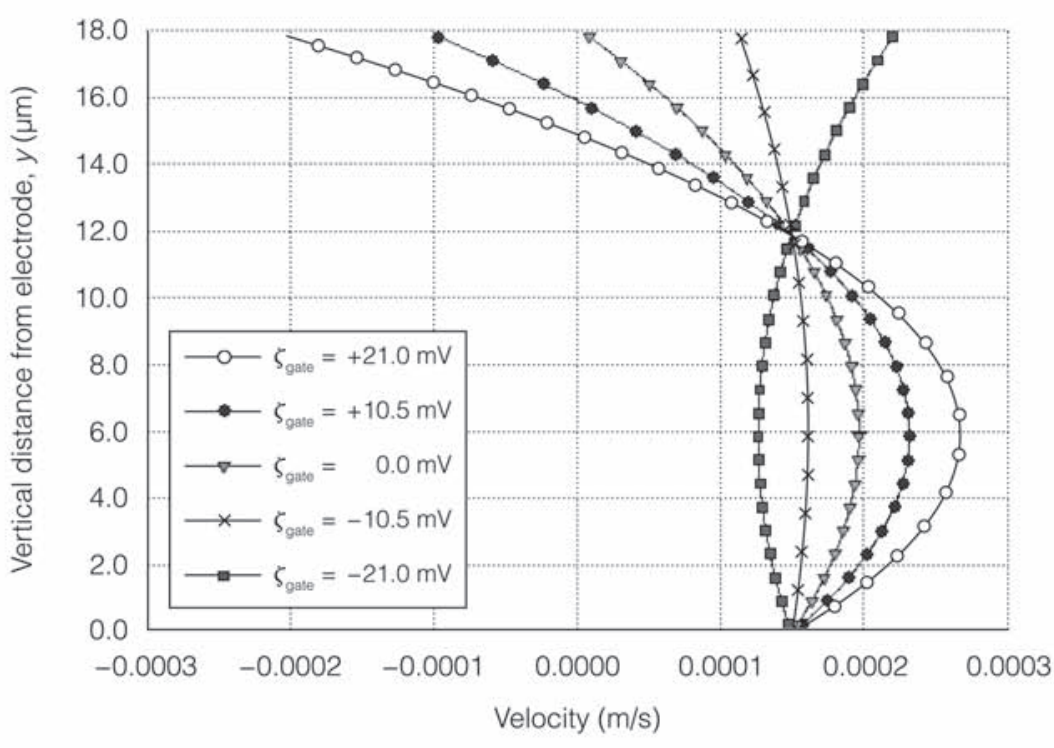

(a)

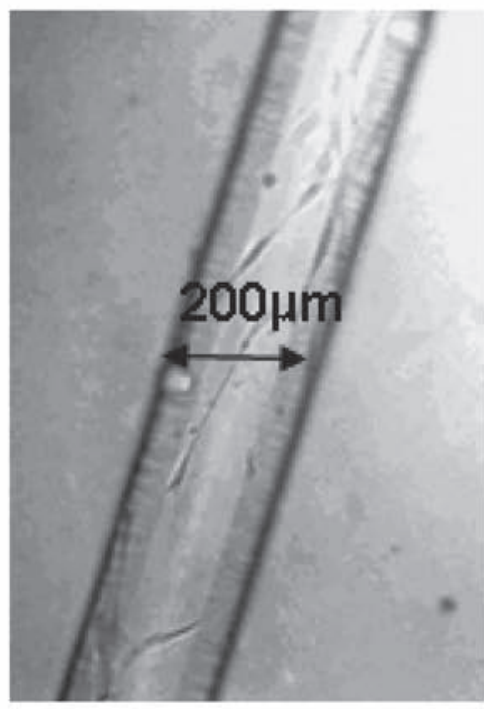

(b)

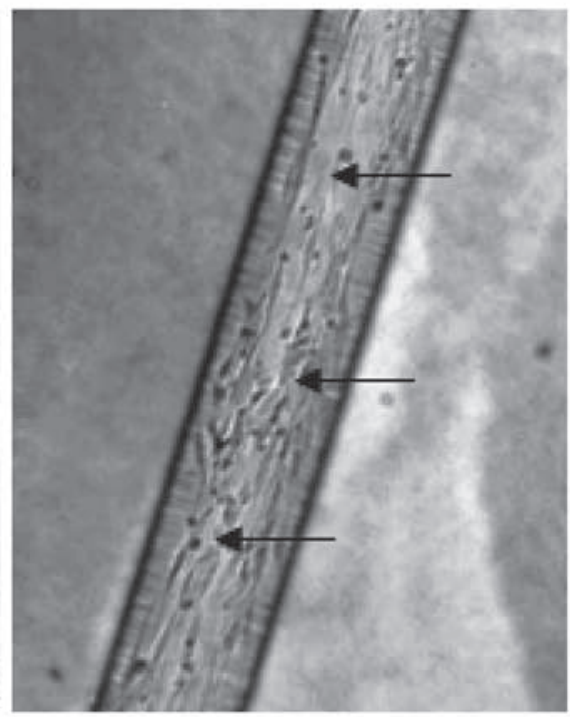

(c)

Figure 3. Fluid velocity in a FlowFET versus vertical distance from the electrode, with zeta potential as the parameter (a), photomicrograph of a fluidic channel (200 microns wide) containing a biofluid (b), and the same channel clogged by bacteria in the biofluid after some time (c).

these simulations. Figure 3a shows fluid velocity and its location under an electrode as functions of the zeta potential. The electrode voltage can change this potential, which also depends on the oxide thickness. Because of the linear relationship, a change in oxide thickness can also cause a change in zeta potential. As Figure $3 \mathrm{a}$ shows, velocity will change as a result, making biochemical reactions, for example, invalid.
Hence, testing these devices means verifying their velocity behavior.

To carry out fluidic routing for a host of applications, designers can connect flowFETs in 2D arrays based on fluidiccrossing modules. Designers can optimize flowFETs with FEM tools, but there is no need for sophisticated high-level design tools. If these MEF arrays contain biological fluids, then very specific, time-dependent defects not found in conventional electronics can occur. In some applications that use a biodevice, disposal of the device in the case of defects is unacceptable. An example is an industrial platform for manufacturing biomaterials such as peptides.

Time-dependent defects in fluids are not a new phenomenon. In ink-jet MEMS, time-dependent contamination of ink particles in the nozzle, which jeopardizes printer dependability, is well known. In biofluids, however, different time-dependent issues play a role. In practice, users have observed the partial or complete jamming of 200micron-wide by 50 -micron-deep channels by growing biomaterial. A peptide environment, for example, is susceptible to bacterial growth. Figure 3b shows a photomicrograph of a fluidic channel containing a biofluid. Figure $3 \mathrm{~b}$ shows the same channel after some time, clogged by growing bacteria. Fluidic crossings, inputs and outputs, often present in large MEFs, are preferred locations of these defects. Kerkhoff et al. provide extensive treatment of this type of defect. ${ }^{8}$

Similar to analog-circuit simulators, the simulation tools serve to verify and optimize the behavior of devices. For example, Figure $4 \mathrm{a}$ shows the simulation result of a fluidic crossing optimized to minimize parasitic fluid recirculation. We performed this simulation by manipulating the zeta potential of the walls.

Designers can subsequently transform FEM simulations into a model at a higher abstraction level-for instance, in a VHDL-AMS (analog/mixed-signal extension of VHDL) environment for fault simulation of 


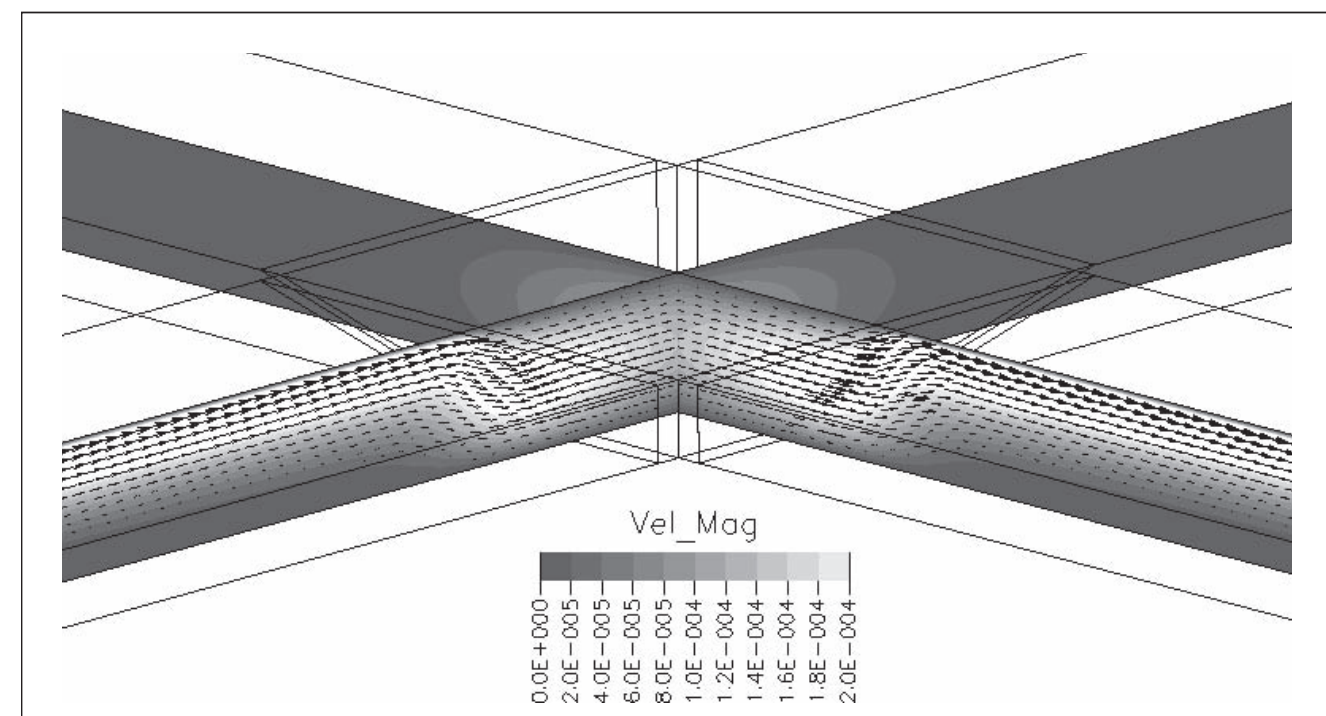

(a)

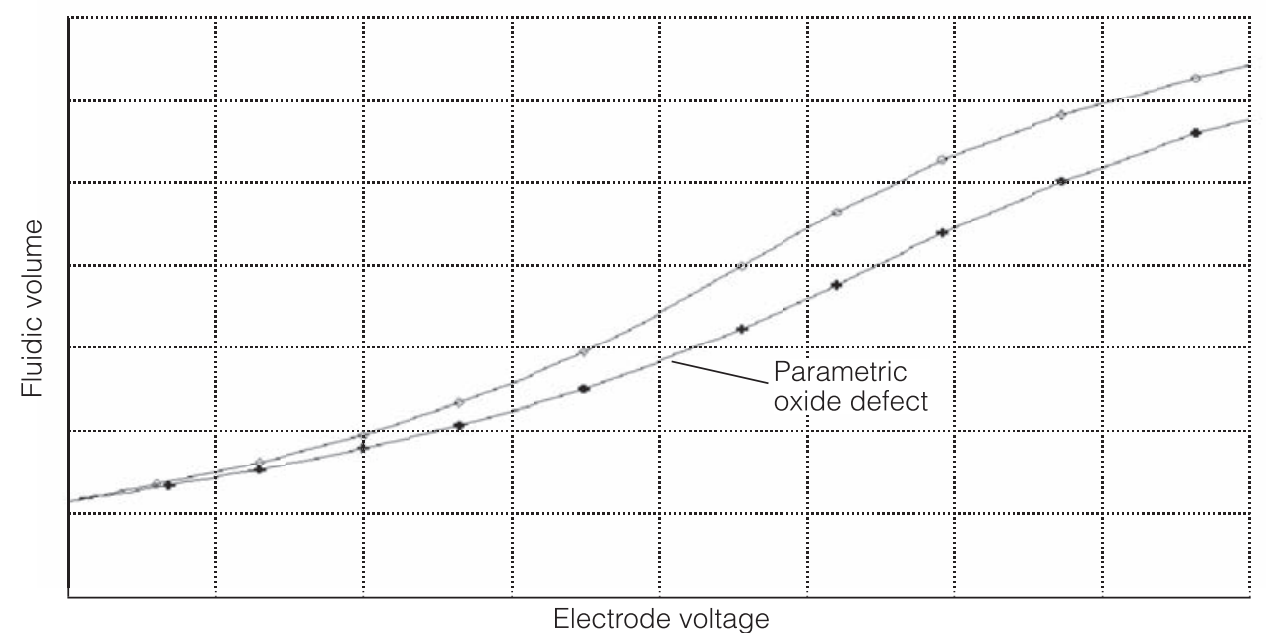

(b)

Figure 4. FEM simulation of fluidic crossing with flowFETs switched to achieve right-hand flow with coating optimization (a), and VHDL-AMS modeling of a flowFET, including a parametric oxide defect in the bottom graph (b).

complex systems. ${ }^{2}$ This approach closely resembles current MEMS-based simulation approaches. Another approach is to use a circuit representation, possibly even enhanced with device models, to incorporate certain physical phenomena. Still another method is a compact modeling approach that models fluidic modules as electrohydraulic conductances and fluidic capacitances; ${ }^{9}$ this method resembles Spice fault simulations.

Figure $4 \mathrm{~b}$ shows the results of a VHDL-AMS simulation of a flowFET, distilled from extensive FEM simulations. The graph shows transported fluidic volume (vertical) versus gate voltage (horizontal), with oxide thickness as a parameter ( $\pm 20 \%)$. Oxide thickness variations clearly influence transport velocity. (Because the channel parameters stay the same, the only way the volume can increase is by a higher velocity.)

VHDL-AMS simulation of fluid velocities and fluid volumes are not the most efficient approach to deal with this problem. Using pressure as a key quantity seems to be more appropriate, and pressure can be readily extracted from FEM simulations.

Now, higher-level fault modeling of the fluidic device is combined with established fault models for 
microelectronics to enable a joint VHDL-AMS fault simulation (cosimulation) of an entire biological MEF array. Using this unified approach, it's possible to investigate the influence of faults in the fluidic as well as the microelectronic domains for the entire MEF system. This enables the development and evaluation of DFT (design for test) constructions and guidance for ATPG algorithms. The DFT constructions in the fluidic domain can be simple flow sensors or capacitive volume sensors in reaction chambers.

The time-dependent biodefects are modeled as a time-dependent hydrodynamic resistance parameter $W_{x}{ }^{10}$ The index $x$ represents the defect's segment location; it can be inserted in each channel, and thus resembles analog-resistive faults in interconnection lines in electronic fault modeling.

At the University of Twente's Centre of Telematics and Information Technology (CTIT), we use the Mentor Graphics ADVance MS tool for fault simulations. The tool is embedded in our $\mathrm{SoC}$ (Cadencebased) CAD environment. As an example, we have simulated a simple MEF bioarray, with four input reservoirs and four output reaction chambers. From the master clock, we generated and simulated all other control voltages for flowFETs through VHDL CMOS gate-level library cells and a Spice-based DMOS model. ${ }^{2}$ Figure 5a shows the results of simulating the array to complete the addition of biofluids from selected sources into a specific reaction chamber. The top three parts of the chart show the clock signal used, the control voltages in volts (the reset pulse), and data for selection of transfer devices. The next signal is the simulated output voltage for DMOST 1. We used Spice models from Philips (bs170) for this purpose. The next signal shows the relevant pressure differences in a selected transfer flowFET (no. 1) and the associated flow in segment 1 . The last signal is the contents of the reaction chamber, which is, as we expected, the accumulated fluid volumes from the selected sources. From FEM simulations, we calculated a biodefect's hydrodynamic resistances and performed fault simulations.

By changing the electrode pulses' durations, as well as their voltage levels, and assuming a capacitive observation point at the reaction chambers, we determined the location (at segment resolution) of defects. The search mechanisms somewhat resemble the algorithms for finding delay faults in critical paths. Also, the input pressure of a fluid, as well as its dynamic variation, can help find potential defects. For instance, a very low pressure will not find jamming particles. The effects of jamming particles and the best solutions for detecting those effects can be found through multiple high-level simulation. ${ }^{2}$ Figure 5b shows a typical setup for testing these arrays. Because flowFETs are analog devices, their testing somewhat resembles functional testing in analog electronics.

FlowFETs can be integrated atop a SoC at relatively low cost (Figure 1 shows an example). However, required high source-drain voltages, which increase linearly with array size, limit the size of current arrays.

\section{Digital microfluidics}

Test developments in digital microfluidics concern different themes of interest from those of analog microfluidics. In the digital domain, there is less concern for test signal generation and fault simulation to enable DFT localization and evaluation. Instead, attention focuses on efficient algorithms for reducing test time and enabling diagnosis for reconfiguration.

The alternative method of manipulating small amounts of fluids avoids closed channels and uses discrete droplets, which contain the conductive biofluid of interest. Although several physical-manipulation mechanisms have proven effective, the most promising one seems to be the electrical-control technique. In this technique, the droplets usually move in a filler medium such as silicone oil and can be controlled individually; hence, the control electrode's topological infrastructure determines their possible paths. This approach is called digital microfluidics.

As Figure 6a shows, the technology strongly resembles that of a flowFET, except for the top electrode and a different type of channel coating. The proper pulsing of electrodes-for example, through the EWOD principle-conveniently moves the droplets. This principle is based on electrostatic manipulation of a droplet's surface tension angle. Droplets slightly overlap the electrodes and move when the central electrode is switched off while an adjacent electrode is switched on. Elementary droplet operations include dispensing, transport, storage (electrode switched off), mixing (by merging droplets and moving them around pivots), splitting, and detection. Figure 6b shows several droplets in an array. Engineers can use videos to measure droplet velocities or observe the influence of defects. Digital microfluidics offers reconfigurability and scalability for complex MEF systems and is less sensitive to tolerances than analog microfluidics. 


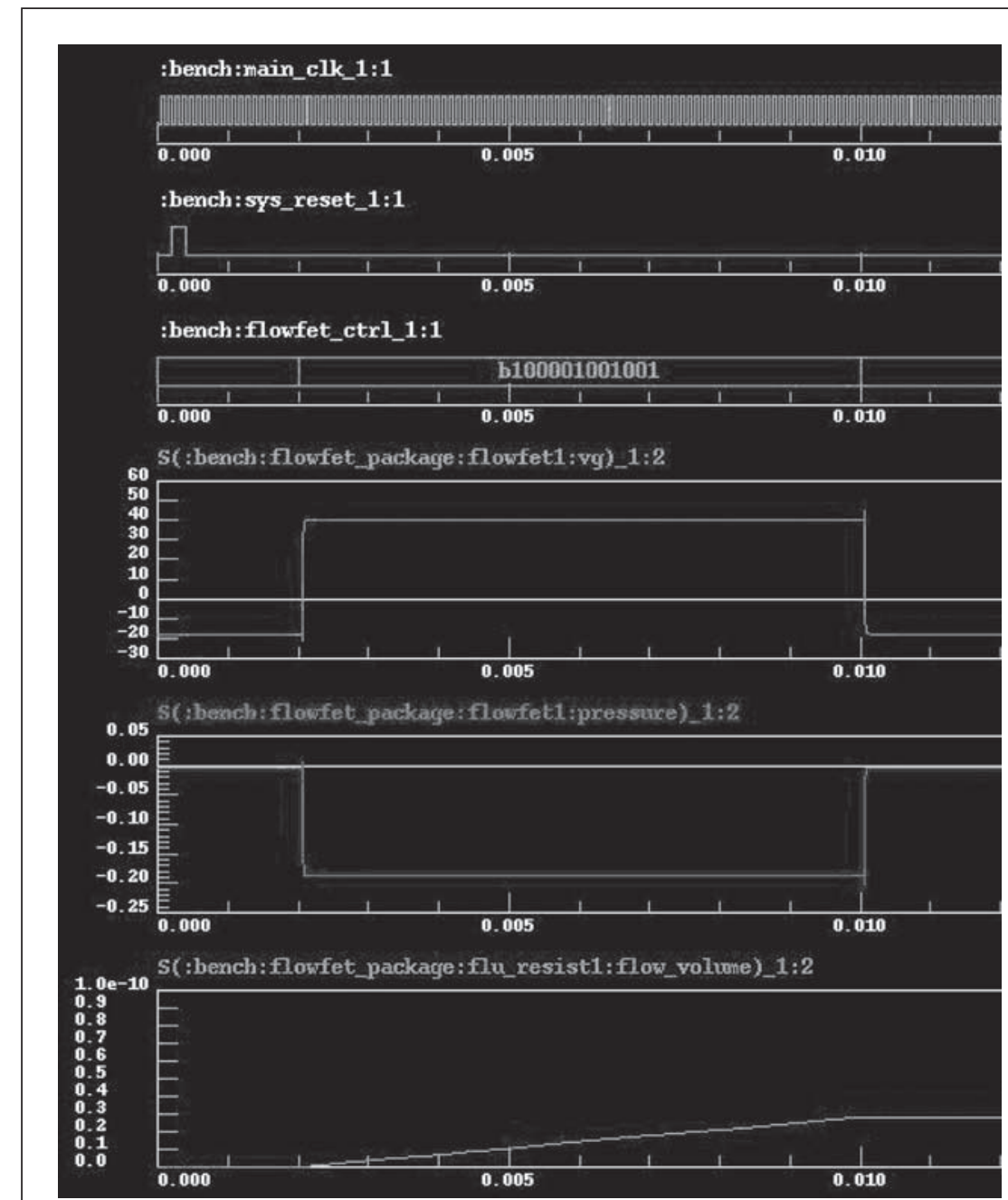

(a)

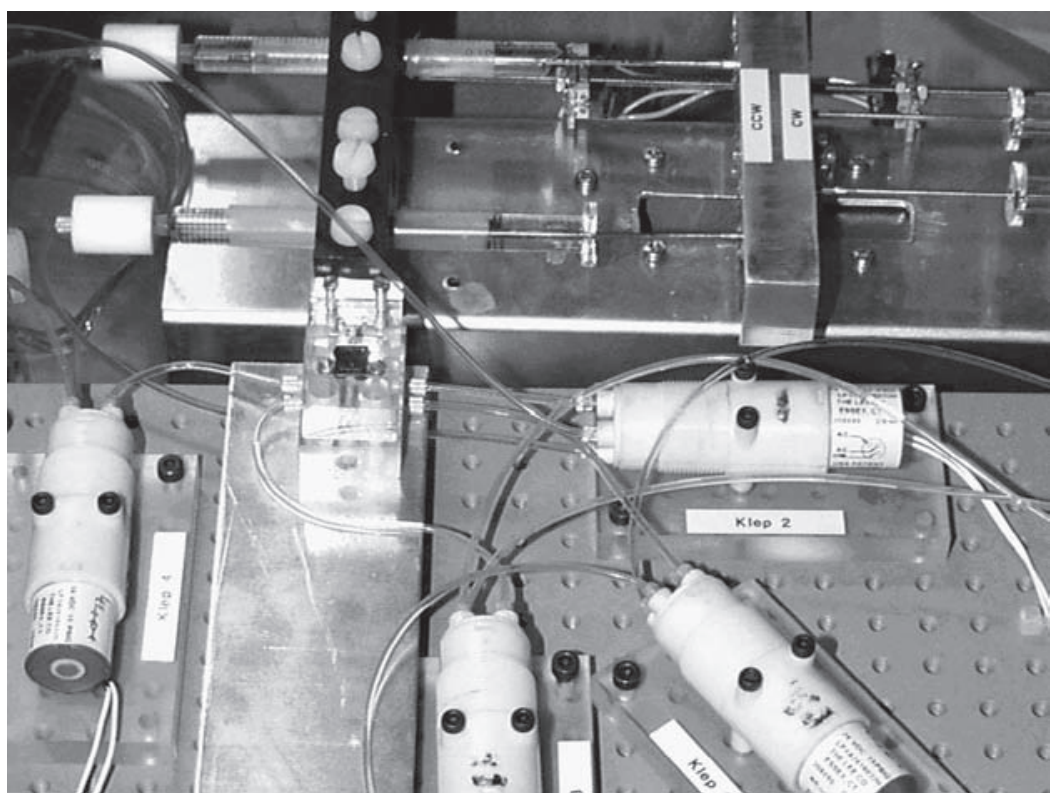

(b)
Figure 5. Dual-domain electricalfluidic VHDL-AMS simulation of a 2 by 2 flowFET array including control electronics (a), and typical test setup for analog microfluidic chips (b).

The physical relationships between droplet velocity during transport and various physical and geometrical parameters are known and predictable for lower voltage ranges (less than $80 \mathrm{~V})$. Because the technology is similar to that of flowFETs, many catastrophic and parametric defects are also similar, such as electrode shorts and opens, insulator and channel-jamming problems, and so forth. Figure $7 \mathrm{a}$ shows an example of how a parametric variation in insulator thickness influences droplet velocity. ${ }^{11}$ An example of a catastrophic defect is an oxide layer breakdown of high-voltage electrodes. This defect results in no charge storage, and therefore electrowetting doesn't occur, hence disabling fluidic transport. If adjacent electrodes are shorted, the droplet is not large enough to overlap the gap between adjacent electrodes, so again a catastrophic fault will occur. However, experiments with shorted electrodes indicated that the orientation of the droplet flow makes a difference on the manifesting behavior, as Figure $7 \mathrm{~b}$ shows. 


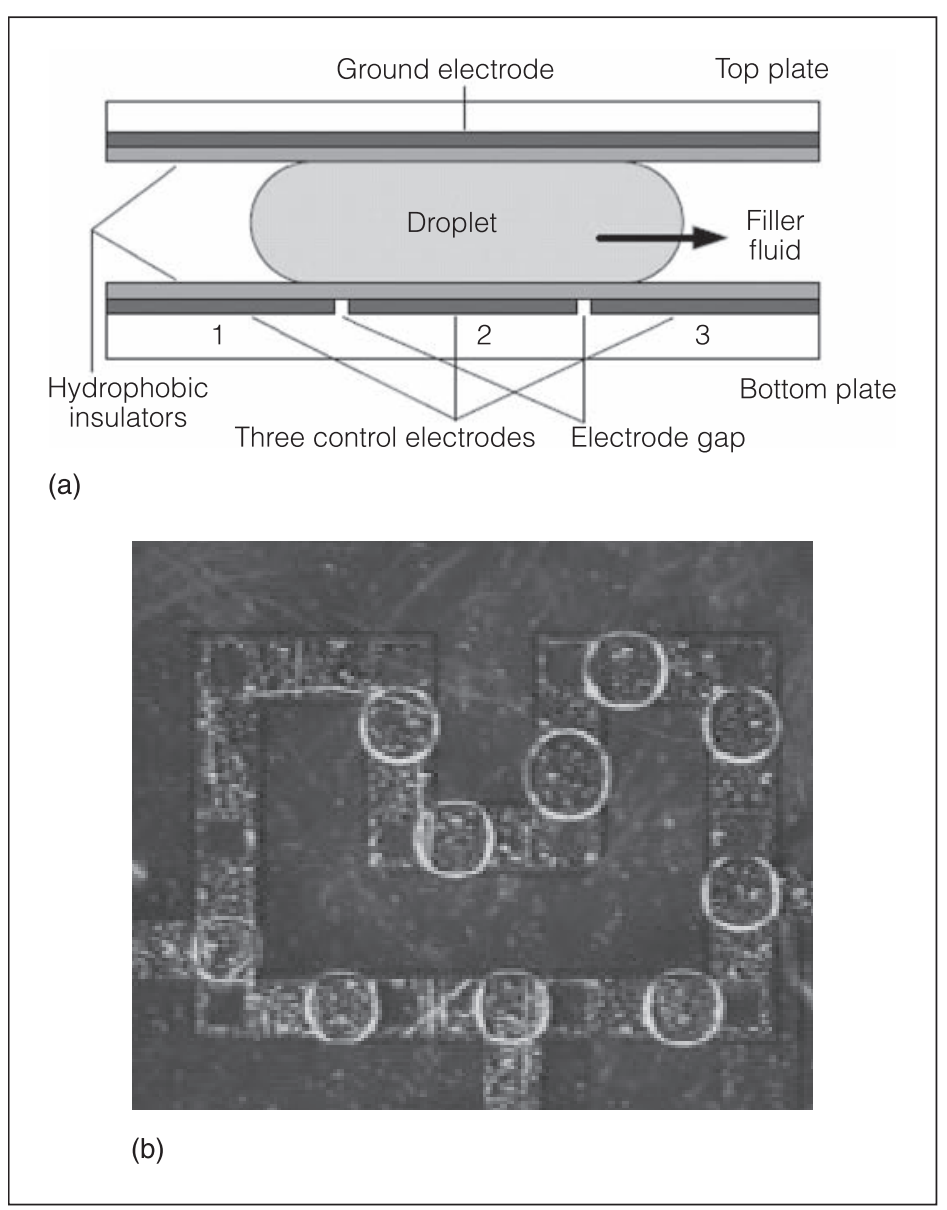

Figure 6. Cross section of a droplet cell and technology (a), and top view of several droplets in an array (b). ${ }^{3-6}$

If the test droplet flow is horizontal, shorted electrodes along this direction will cause the droplet to stick under the shorted gates. If the shorted electrodes are in the $y$ direction, and the droplet flow is in the $x$ direction, the droplet will not stick and will continue its transport. Hence, an error results only if the droplet flow is aligned with the shorted electrodes. This unexpected behavior holds for other defects, such as particle contamination between gates. ${ }^{11,12}$ So, for detecting edge-related defects, testing must consider not only single droplet cells but also droplet pairs and droplet traversal to all their neighbors. This calls for tailored test algorithms.

Testing digital microfluidics basically means monitoring the droplet's velocity or presence at a boundary cell. $^{3}$ Sensing the change of capacitance under an electrode at the arrival of a droplet can conveniently perform this observation. Combining this capability with a test-droplet-dispensing element (generator) results in a very compact DFT structure. By changing the direction and duration of control pulses, you can determine the lower and upper bound droplet velocities. Similar to the flowFET, optical observation with a camera is also possible. From this observation, you can determine the speed of droplets, and it is very useful to study mixing and splitting techniques of droplets by looking at their contours. Imaging software has been developed to extract these contours. In addition, droplet presence and droplet contents can be evaluated by means of on-board (local) photo sensors. Direct defect-oriented droplet testing has not been thoroughly investigated; hence, the approach is fundamentally different, as in the case of flowFETs. The small number of industrial droplet implementations are tested as in the analog-microfluidics approach or by linking to functional tests.

Test coverage for digital microfluidics means that all cells are available for testing (except active cells). Test optimization uses the time slot concept; a time slot is the time required for a droplet to transfer from one location to an adjacent location. In single-droplet testing, test time is the time required for all cells to be visited by a droplet; but, as mentioned before, this test time must be extended to droplet pairs and droplet transversal.

Initially, testing didn't consider droplet pairs and droplet traversal. The resulting scheduling problem is NP complete, but integer linear programming can completely solve the problem. ILP works for multiple droplet resources and can include safety margins to avoid accidental mixing of test and functional droplets in concurrent testing. Unfortunately, ILP is a timeintensive approach that is unsuitable for large arrays. You can reduce the time for large arrays by partitioning them into nonoverlapping smaller arrays.

Alternatively, you can use heuristics-for example, the easily implemented scan-path-based algorithm. The disadvantage of this algorithm is that the waiting time for functional droplets can be excessively long, and hence the test time is too long. Researchers have developed new heuristics based on motion-planning optimization of moving robots. One example is the simple Monte Carlo search algorithm; another is the modified real-time algorithm. In general, these heuristics provide test plan solutions close to the lowerbound optimal test time.

All previous algorithms considered only single cells. Researchers have now developed new algorithms to account for droplet pairs and droplet traversal to all 
neighbors. To enhance testing support capabilities, these algorithms also take into account the automatic insertion of DFT in terms of the most favorable test droplet source, and sink and observation locations. To include defects that depend on the orientation of the droplet (Figure 7b), the test plan is formulated as an Euler circuit solution using graph theory. As Figure 8 shows, this approach models the droplet array as an undirected graph with vertices and edges and then "eulerizes" the graph. An Euler circuit is a cycle that traverses all edges (interconnections between vertices) of the graph exactly once, with each vertex having an even degree (even number of edges). Most microfluidic graphs have more than two vertices of odd degree (Figure 8b).

To satisfy the rules for an Euler circuit, you must convert a microfluidic-array graph by applying Euler's theorems. Then you construct a test plan from this converted graph. You convert the microfluidic array graph by adding edges (Figure 8c, upper left, bold arcs). The Euler circuit approach has no limitations on source and sink locations, and there is no need to change the eulerized graph each time a droplet takes a different sink and source location adjacent to a boundary cell. Therefore, these DFT locations can be optimized. If you merge source and sink locations, you can recycle test droplets, thus minimizing DFT hardware. The total test time is the number of edges traversed multiplied by the time a droplet takes to go from one vertex to the adjacent vertex.

To find an Euler circuit in the converted graph, you use the Fleury real-time algorithm. ${ }^{11}$ To reduce computational costs, you apply probabilistic search procedures (probabilistic modified Fleury). In the case of additional test droplets (multiple sources and sinks), you partition the graphs, and each droplet is considered following the previous one in the test droplet flow. You calculate the total test time by adding the separate test times.

In addition to this offline testing, concurrent testing is also possible-that is, simultaneous testing and functional operation-if no conflicts occur between droplets. ${ }^{4}$ For online testing applications, the solution is more complex. Effectively, waiting times for test droplets increase testing time because of temporarily unavailable cells required for functional operation.

Dependable microfluidic systems such as healthand environmental-monitoring systems require defect diagnosis in addition to testing-that is, detection of the location and nature of defects. After defect

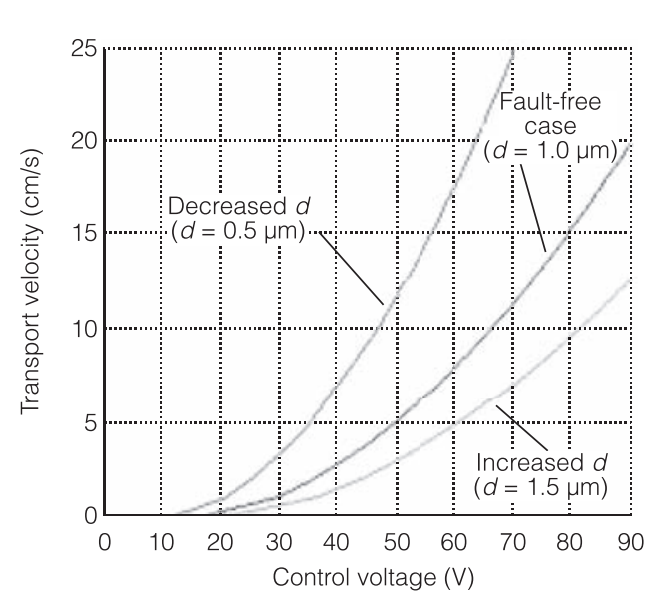

(a)

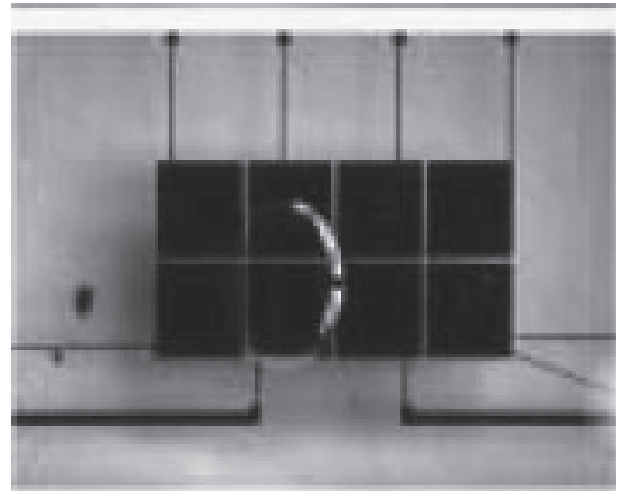

(b)

Figure 7. Influence of dielectric variation on droplet velocity (where $d$ is dielectric layer thickness in microns) (a), and transfer of the droplet despite a vertical, adjacent electrode short (b). ${ }^{11}$

diagnosis, spare cells can be applied to reconfigure faulty parts. Defect diagnosis takes an Euler-based approach using multiple test steps. The technique uses recursive binary partitioning (as Figure $8 \mathrm{c}$ shows), determining the number of steps by the array dimensions. The total diagnosis time is the sum of the four test times of the test step parts ( $T_{i}$ in Figure $8 \mathrm{c}$ ), which is 68 in Figure 8. The approach is valid for single as well as multiple fault assumptions and multiple test droplets, but the multiple conditions require some algorithm adaptations. If $T_{i}=1$, the defect has been detected in the partition-for example, in the first test partitioning step (Figure 8c, upper left). Otherwise, $T_{i}=0$ as in the upper right case (second test partitioning step). From this data, the $T_{i}$ values and different paths can determine the defect location. Next, on the basis of 


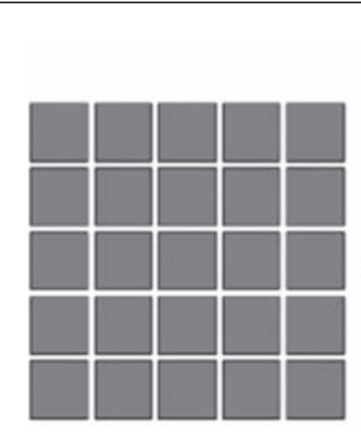

(a)

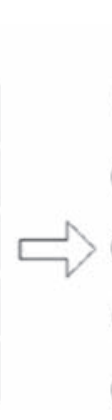

(b)
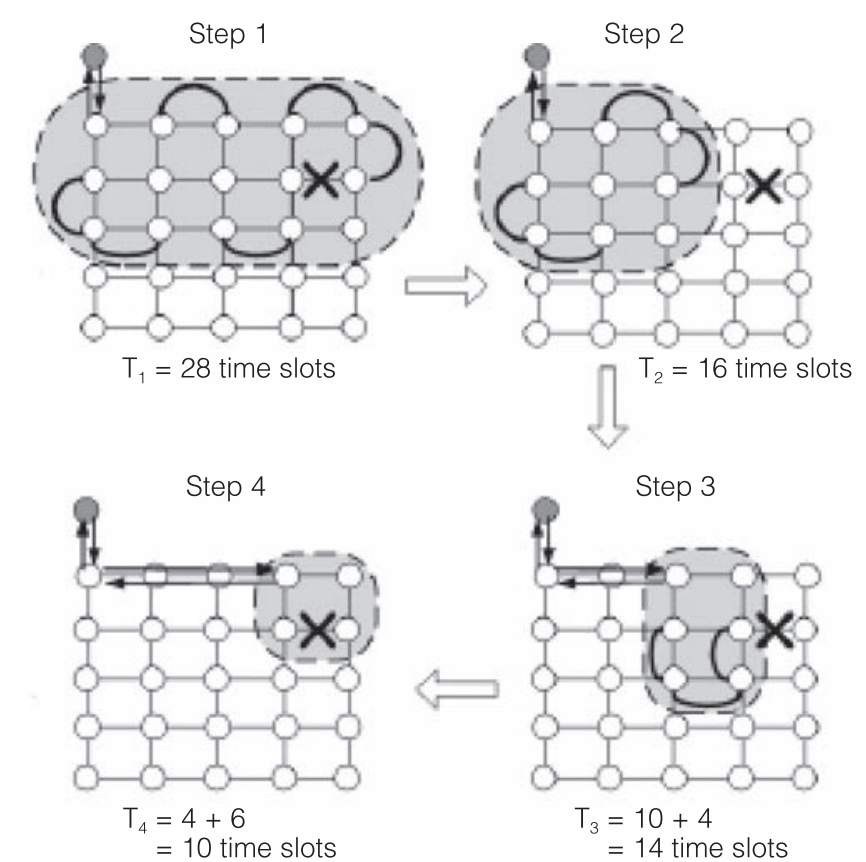

Test droplet source or sink

(c)

Figure 8. Representation of microfluidic array (a), conversion to undirected graph (b), and diagnosis of defect by multistep test partitioning (c). Here, $T_{1}$ through $T_{4}$ are the test times for the steps in the test partitioning process. The total diagnosis time is the sum of the four partitioning times (68 time slots).

the defect location, reconfiguration of modules, such as mixers, can take place.

The placement of fluidic modules in a digitalmicrofluidic array is important, especially for allowing (partial) dynamic reconfigurability in the case of faulty cells. You implement reconfigurability by using additional (spare) electrodes in the core area and by reusing the original module's voltage pulses provided

by a microcontroller. Figure 9 a shows a $6 \times 6$ cell core area, which contains three modules (such as mixers). The previously described algorithm shows one cell to be faulty (due to an insulator defect, for example). Figure $9 \mathrm{~b}$ shows a possible reconfiguration solution, which neatly isolates the faulty cell.

Because placement is an NP-complete problem, you can solve it by using the simulated annealing concept, well known in the past for placing functional blocks in microelectronics. You can introduce a fault tolerance index (FTI) between 0 (no reconfigurability possible) and 1 (full reconfigurability) to estimate the system's fault tolerance capability. Obviously, the fault tolerance capability depends on placement.

The following is the complete procedure for designing digital MEF arrays: First, represent the various operations (such as mixing) and fluids involved, as well as their mutual relationships, in a sequencing graph. Assuming that the times required for the operations and fluids involved are known, derive a time-scheduling scheme. In some cases, certain basic modules are active only during certain time slots. This enables module reuse through reconfigurability, so there are fewer modules to place. This can reduce placing and timing from a $3 \mathrm{D}$ to a pseudo-2D problem. Second, carry out placement, with modules having the largest area placed first; add a wrapper of one electrode around these modules to avoid interference and allow droplet transfer. Then, perform simulated annealing. Third, to find out which spare area is available in the resulting array, find the emptiest rectangles (which equal unused electrodes), and then check whether they can accommodate a faulty module. Fourth, to increase the system's fault tolerance, guide placement by a weighted sum, including the required FTI and area. After performing area minimization, increase the FTI. In this way, the designer can make trade-offs between fault tolerance and area consumption.

Figure 9c illustrates the placement results for seven modules. The time slots in which the different modules are active are shown on the left, and the final placement, including spare cells to guarantee the required FTI, is shown on the lower right.

INTEGRATING HETEROGENEOUS SYSTEMS, sUCh as combining fluidics with microelectronics in MEF arrays, is growing in importance. However, the design and test of MEF arrays is still immature because 


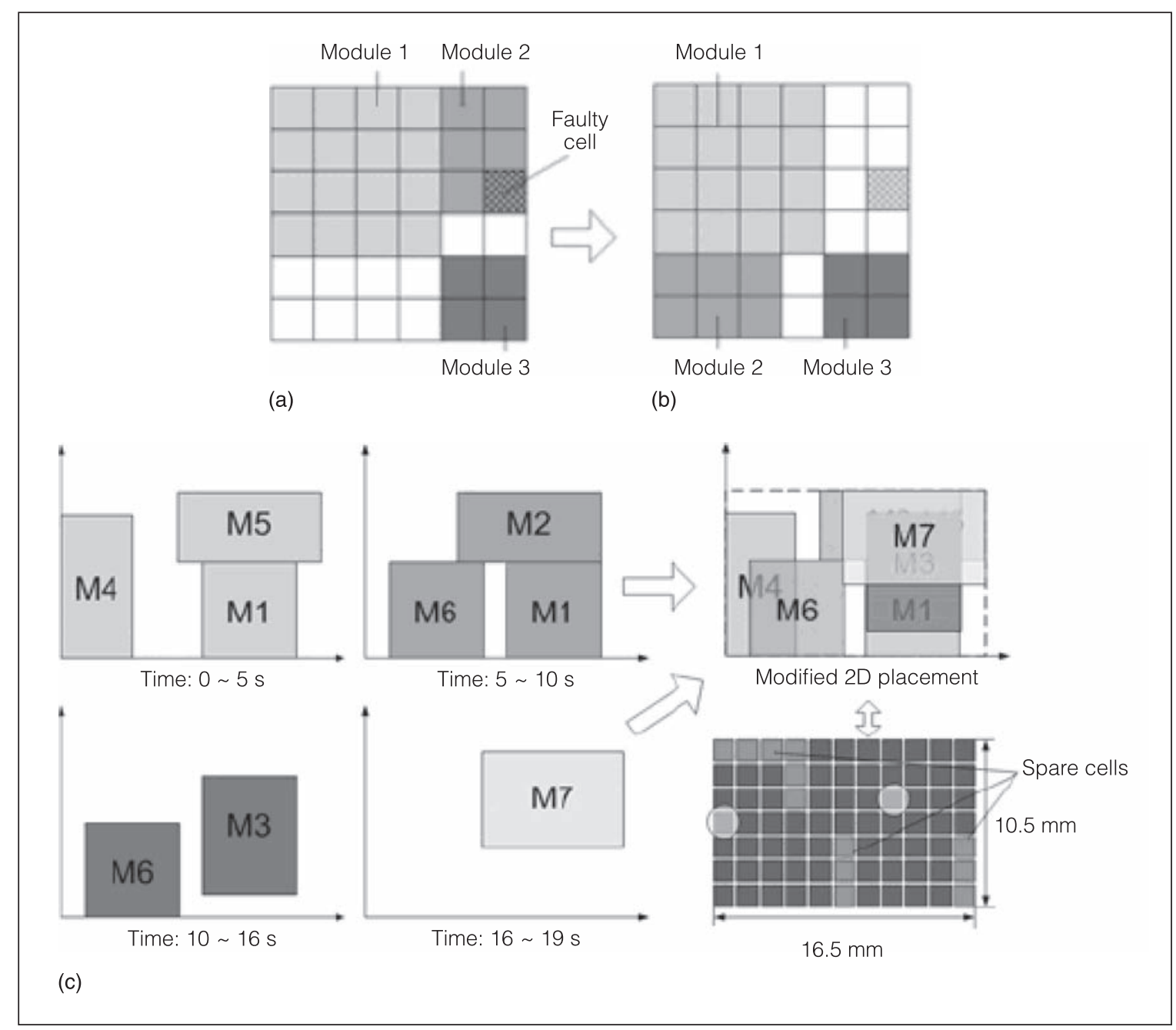

Figure 9. Faulty module including faulty cell (a), subsequent possible reconfiguration (b), and automatic placement taking reuse into account, including spare cells for reconfiguration (c). 5

designers and test engineers lack tools and experience. Unfortunately, the implementations of most biological applications are disposable after first use, so many companies don't take the testing of these devices seriously. At the same time, research in analog and digital microfluidics is meeting new design and test challenges. Defect-oriented testing, as well as the development of DFT and offline and online test algorithms, show that quality can be measured and hence improved. Thus, the commercial road to a variety of exciting microelectronic biofluidic applications is wide open.

\section{References}

1. H.G. Kerkhoff, "Testing Philosophy behind the Micro

Analysis System," Proc. SPIE, Int'I Soc. Optical

Engineering, vol. 3680, 1999, pp. 78-83.
2. H.G. Kerkhoff and M. Acar, "Testable Design and Testing of Micro-Electro-Fluidic Arrays," Proc. 21st VLSI Test Symp. (VTS 03), IEEE CS Press, 2003, pp. 403-409.

3. F. Su, S. Ozev, and K. Chakrabarty, "Testing of DropletBased Microelectrofluidic Systems," Proc. Int'l Test Conf. (ITC 03), IEEE CS Press, vol. 1, 2003, pp. 1192-1200.

4. F. Su, S. Ozev, and K. Chakrabarty, "Concurrent Testing of Digital Microfluidics-Based Biochips," Proc. Int'l Test Conf. (ITC 04), IEEE CS Press, 2004, pp. 883-892.

5. F. Su and K. Chakrabarty, "Design of Fault-Tolerant and Dynamically Reconfigurable Microfluidic Biochips," Proc. Design, Automation and Test in Europe (DATE 05), IEEE CS Press, 2005, pp. 1202-1207.

6. K. Chakrabarty, "Design, Testing and Applications of Digital Microfluidics-Based Biochips," Proc. 18th Int'l Conf. VLSI Design (VLSID 05), IEEE CS Press, 2005, pp. 221-226. 
7. J. Gong, S.-K. Fan, and C.J. Kim, "Portable Digital Microfluidics Platform with Active but Disposable Lab-onChip," Proc. 17th Int'l Conf. Micro Electro Mechanical Systems (MEMS 04), IEEE Press, 2004, pp. 355-358.

8. H.G. Kerkhoff et al., "Fault Modelling and Co-simulation in FlowFET-Based Biological Array Systems," Proc. 3rd Int'l Workshop Electronic Design, Test and Applications (DELTA 06), IEEE CS Press, 2006, pp. 177-182.

9. A.N. Chatterjee and N.R. Aluru, "Combined Circuit/ Device Modelling and Simulation of Integrated Microfluidic Systems," IEEE/ASME J.

Microelectromechanical Systems, vol. 14, no. 1, Feb. 2005, pp. 81-95.

10. P. Tabeling, Introduction to Microfluidics, Oxford Press, 2005.

11. F. Su, Synthesis, Testing and Reconfiguration Techniques for Digital Microfluidic BioChips, doctoral dissertation, Dept. of Electrical Engineering and Computer Science, Duke Univ., 2006.

12. F. Su et al., "Defect-Oriented Testing and Diagnosis of Digital Microfluidic-Based Biochips," Proc. Int'l Test Conf. (ITC 05), IEEE CS Press, 2005, pp. 487-496.

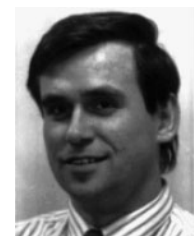

Hans G. Kerkhoff is an associate professor and head of the Testable Design and Testing of Integrated Systems Group at the Centre of Telematics and Information Technology (CTIT), a subsidiary of the University of Twente, the Netherlands. He is the founder of the test consultancy company TwenTest. Kerkhoff has an MS in telecommunication from the Technical University of Delft and a PhD in technical science from the University of Twente.

Direct questions and comments about this article to Hans G Kerkhoff, CTIT, Testable Design and Testing of Integrated Systems Group, Dept. of EEMCS, Hogekamp 45, University of Twente, Post Box 217, 7500AE Enschede, the Netherlands; h.g.kerkhoff@ utwente.nl.

For further information on this or any other computing topic, visit our Digital Library at http://www.computer.org/ publications/dlib

\section{Here Now! \\ Introduction to Python for Artificial Intelligence}

By Steven L. Tanimoto University of Washington

Python, an increasingly popular general-purpose programming language, offers a variety of features that make it especially well-suited for artificial intelligence applications. This ReadyNote will help professional programmers pick up new skills in Al prototyping and will introduce students to Python's Al capabilities. $\$ 19$

www.computer.org/ReadyNotes

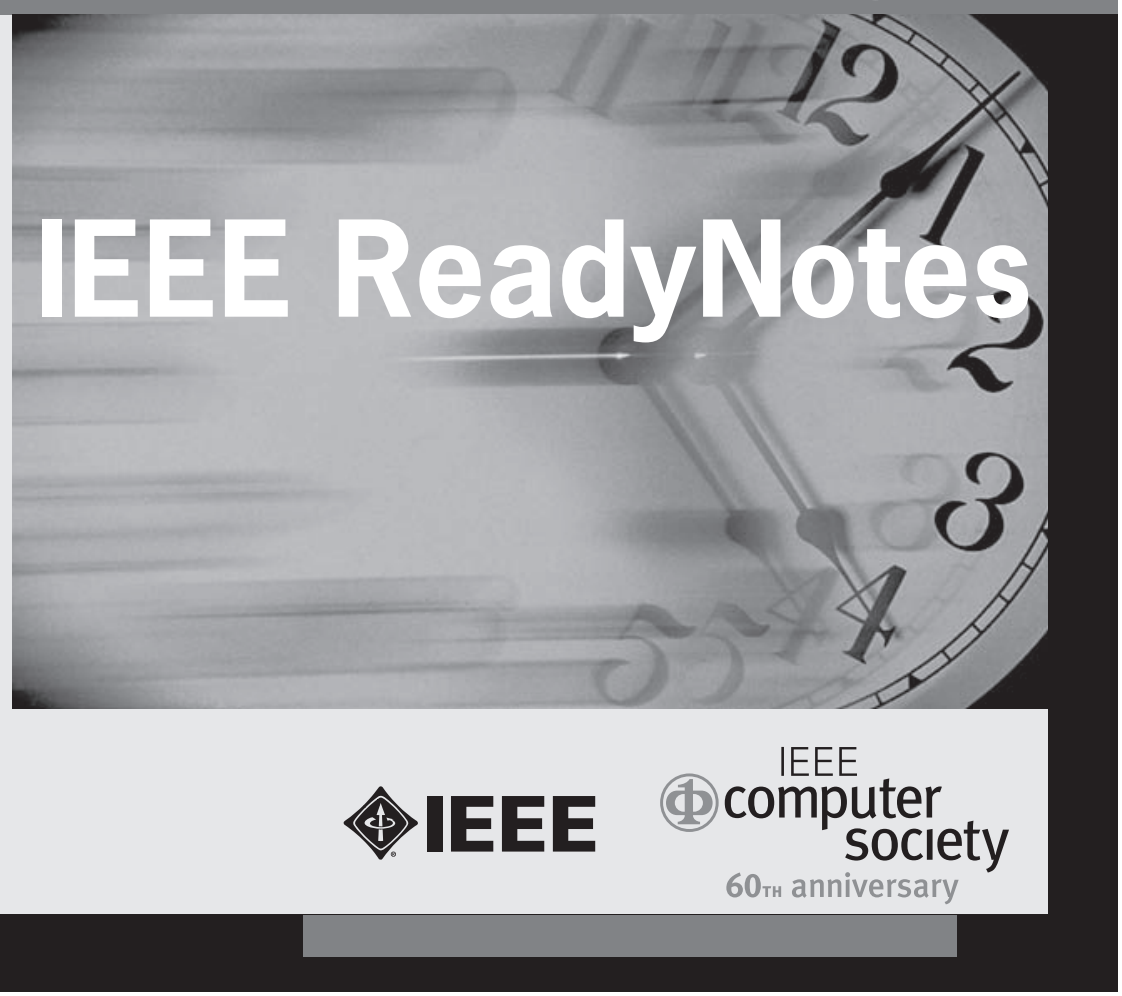

